\title{
Pretraining performance of three species of macaque monkeys'
}

\begin{abstract}
Abstraet
Rhesus (Macaca mulatta), Philippine cynomolgus (M. irus), and stump-tailed(M. speciosa)macaques were given the same 16-step pretraining procedure in a Wisconsin General Test Apparatus (WGTA). The stumptailed monkeys completed pretraining in significantly fewer trials than did the rhesus and Philippine monkeys. The pretraining performance of the latter two species was about the same.
\end{abstract}

\section{Problem}

Much behavioral work has been done on the rhesus macaque, but very little on the Philippine cynomolgus and stump-tailed macaques (Schrier, Harlow, \& Stollnitz, 1965). Observations of these monkeys in their cages and during general handling reveal a number of differences in behavior, but the stump-tailed monkeys are of special interest because they seem to be unusually docile, mild-mannered animals (Kling \& Orbach, 1963; Orbach \& Kling, 1964). In contrast to rhesus and Philippine cynomolgus monkeys, stumptailed monkeys can be easily handled and restrained without special training. Furthermore, informal observations at the Brown University Primate Behavior Laboratory and elsewhere ${ }^{2}$ indicate that the stumptailed monkey adapts to new experimental situations more readily than do the other two types of macaques. The purpose of the present study was to formalize such observations by measuring the rate of progress of the three species in pretraining in a WGTA. Method

The Ss were 14 Philippine cynomolgus, 15 stumptailed, and 8 rhesus macaque monkeys (respectively, Macaca irus, M. speciosa, and M. mulatta). The animals in each group were between 3 and 4.5 years old, as judged from the number of erupted permanent teeth. There were roughly equal numbers of males and females in each group and all were experimentally naive. Before the experiment, the animals had been in the laboratory for 2 to 3 months, during which they had been trained to enter small transfer cages. Whole-diet biscuits and, later, sugar pellets were used as reinforcers for this transfer-cage training.

The apparatus, the Brown University WGTA, has been described in detail before (Schrier, 1961). Briefly, it consists of a cage for $S$, a test tray, and three movable screens as follows: An opaque screen, between the cage and the test tray, prevents $S$ from viewing the test tray while it is being prepared for a trial. A transparent screen, about 1 in behind the opaque screen, prevents $\mathrm{S}$ from responding before the opaque screen has been raised fully. A one-way-vision screen, between $\mathrm{E}$ and the test tray, prevents $\mathrm{S}$ from observing $\mathrm{E}$ during a trial. The test tray contained two cup-shaped foodwells about $1-1 / 8$ in in diameter, $1 / 4$ in deep and 12 in apart center to center. The distance from the front bars of the cage to the centers of the foodwells was about 9 in. The manipulanda were a gray block, 3 in square and $5 / 8$ in high, and eight junk objects drawn at random from the large collection kept in the laboratory. All but one of the junk objects was mounted on a gray block of the same dimensions as the one just described. The manipulanda could be chained to the WGTA to prevent Ss from pulling them into the cage. The reinforcers were 45-mg sucrose pellets (P. J. Noyes).

The Ss were given 20 trials a day in the WGTA, five days a week. The intertrial interval (ITI) was $10 \mathrm{sec}$. On each trial, one of the two fooiwells was baited with a sugar pellet. The left and right foodwells were baited in random order with the restriction that they be used equally often every 10 trials. A trial ended when $S$ either took the sugar pellet or balked, that is, failed to take the pellet within 20 sec. Pretraining, appropriate in this case for two-choice objectdiscrimination learning, consisted of 16 steps. A step was continued until either of the following criteria was met: (a) $S$ took the sugar pellet within $20 \mathrm{sec} . \mathrm{k}$ trials in a row, in which case the next step was begun, or (b) $\mathrm{S}$ balked $\mathrm{k}$ trials in a row, in which case the previous step was repeated.

Each step added an aspect of the full WGTA procedure as follows: Step $1(k=20)$. All three screens were kept in the up position, out of use. To begin each trial, $\mathrm{E}$ showed $S$ the sugar pellet and then placed it in one of the two foodwells. If $S$ balked, $E$ ended the trial by removing the pellet. Step $2(\mathrm{k}=10)$. The transparent screen was in the down position during ITI. The foodwell was baited as in Step 1. The transparent screen was raised to begin a trial and lowered to end it. Step 3 $(\mathrm{k}=10)$. The opaque screen, in addition to the transparent screen, was in the down position during ITI. The foodwell was now baited during ITI. The opaque screen was raised, then the transparent, to begin a trial, and both screens were lowered simultaneously to end it. Step $4(\mathrm{k}=5)$. The one-way-vision screen was lowered at the end of ITI, so that S could no longer see E during a test session. ${ }^{3}$ The gray block was placed about $1 / 4$ in behind the baited foodwell. Step $5(k=5)$. The gray block covered the rear quarter of the baited foodwell. Step $6(k=5)$. The gray block covered the rear half of the baited foodwell. Step $7(k=5)$. The gray block 
TABLE I

Mean Number of Trials Taken by the Three Species of Macaques to Complete Each Step or Group of Steps

\begin{tabular}{lcccccccc} 
Species & \multicolumn{8}{c}{ Step } \\
& $1^{\star}$ & $2^{\star \star}$ & $3^{*}$ & 4 & $5-7$ & 8 & $9-16$ & $1-16^{*}$ \\
\hline Rhesus & 76 & 16 & 14 & 7 & 25 & 24 & 42 & 204 \\
Philippines & 62 & 26 & 15 & 11 & 26 & 28 & 46 & 214 \\
Stump-tails & 33 & 11 & 11 & 6 & 22 & 26 & 60 & 169 \\
\hline
\end{tabular}

${ }^{\star}$ Differences between means significant beyond the .05 level

${ }^{\star}$ Differences between means significant beyond the .01 level covered the rear three-quarters of the baited foodwell. It was now practically impossible for $S$ to remove the sugar pellet without displacing the block in the process though the pellet could still be seen easily. Step $8(k=20)$. The gray block completely covered the baited foodwell. This step was always continued to the end of the session in which criterion (a) was met, so that the following step would begin on the first trial of a session. The trials after the criterion trials were not included in the tabulation of results. Step 9 $(k=5)$. The procedure was the same as that of Step 8 except that a junk object was substituted for the gray block and that no trials were given after the criterion trials. Steps 10-16. Step 9 was repeated using a new junk object for each step.

\section{Results and Diseussion}

Table I shows the mean number of trials taken by Ss of each species to complete the individual or related groups of steps and all 16 steps combined. Because of marked heterogeneity of variance, especially of the scores for each of the first three steps, the nonparametric Kruskal-Wallis analysis-of-variance test was used. The means for Steps 1-16 combined differed significantly as did the means for each of the first three steps (see Table I for ps). Thus the first three steps were the primary reason for the significantoverall difference in performance of the three species. The Mann-Whitney $U$ test was used to compare pairs of means in those cases in which the analysis-of-variance test was significant. The stump-tailed monkeys com- pleted each of the first three steps as well as Steps 1-16 combined in significantly fewer trials than did either of the other two species; in none of these cases was the difference in performance of the Philippine and rhesus monkeys reliable.

The results of the present study confirm informal observations. Not only is the stump-tailed monkey gentler and easier to handle than the Philippine macaque or that old standby, the rhesus macaque, but it appears to adapt to new experimental situations more readily. Does this mean that the stump-tailed monkey is the answer to an animal psycholgist's prayers? Perhaps, but some negative features might be worth mentioning. Compared with the other two macaque species studied here, stump-tails vocalize louder and more shrilly and have a considerably more unpleasant odor. Although the stump-tails are on the same diet as the other species, their feces are frequently looser and, as a consequence, their home and test cages are harder to keep clean. However, when frequent handling is required or when pretraining time must be minimized, their advantages far outweigh their disadvantages.

\section{References}

Kling, A., \& Orbach, J. The stump-tailed macaque: a promising laboratory primate. Science, 1963, 139, 45-46.

Orbach, J., \& Kling, A. The stumped-tailed macaque: a docile Asiatic monkey. Anim. Behav., 1964, 12, 343-347.

Schrier, A. M. A modified version of the Wisconsin General Test Apparatus. J. Psychol., 1961, 52, 193-200.

Schrier, A. M., Harlow, H. F., \& Stollnitz, F. (Eds.) Behavior of nonhuman primates. New York: Academic Press, 1965, 2 vols. Notes

1. This study was supported by Research Grant MH-07136 from the National Institute of Mental Health, U. S. Public Health Service. 2. For example, D. Symmes and K. V. Anderson, Yale University School of Medicine, personal communication, 1965.

3. Through a misunderstanding, the one-way-vision screen was not used in pretraining the rhesus monkeys. Neither the present results, nor extensive data from past pretraining of rhesus monkeys using a similar procedure, indicate that the omission had a serious effect on pretraining performance. This is not to suggest that the one-way-vision screen can be dispensed with, for it almost certainly helps prevent $\mathrm{E}$ from giving undesired visual cues to $\mathrm{S}$ during discrimination studies. 